\title{
Quantum dew: Formation of quantum liquid in a nonequilibrium Bose gas
}

\author{
S. Khlebnikov \\ Department of Physics, Purdue University, West Lafayette, Indiana 47907 \\ I. Tkachev \\ Department of Physics, Purdue University, West Lafayette, Indiana 47907, \\ TH Division, CERN, CH-1211 Geneva 23, Switzerland, \\ and Institute for Nuclear Research, Russian Academy of Sciences, Moscow 117312, Russia
}

(Received 9 February 1999; published 24 March 2000)

\begin{abstract}
We consider phase separation in a nonequilibrium Bose gas with an attractive interaction between particles. Using numerical integrations on a lattice, we show that the system evolves into a state that contains drops of a Bose-Einstein condensate suspended in uncondensed gas. When the initial gas is sufficiently rarefied, the rate of formation of this quantum dew scales with the initial density as expected for a process governed by two-particle collisions.
\end{abstract}

PACS number(s): 98.80.Cq, 03.75.Fi

\section{INTRODUCTION}

The theory of interacting Bose gases has been an important part of quantum statistical mechanics ever since Bogoliubov's seminal work [1]. In particular, nonequilibrium Bose gases are interesting from at least two points of view. First, such gases can now be produced in the laboratory via modern cooling techniques. A dramatic demonstration of the resulting nonlinear dynamics is Bose-Einstein condensation (BEC) observed in alkali vapors [2-4]. Second, nonequilibrium gases of elementary particles frequently arise in cosmological scenarios and could have played an important role in the evolution of the universe.

One way how nonequilibrium Bose gases arise in cosmology is via decay of a coherently oscillating field. This mechanism could be important, for instance, at the end of an inflationary stage, i.e., during the reheating after inflation. Indeed, it has been found that in some inflationary models, the oscillating inflaton field decays rapidly and completely into a gas that contains both the inflaton quanta and other types of Bose particles [5]. These gases have very large occupation numbers in low-momentum modes and almost no occupation in high-momentum modes; they are highly nonthermal. The possibility of existence of such gases is by no means limited to the postinflationary era. In particular, there are indications that nonbaryonic cold dark matter constitutes a significant fraction of the matter in the universe at present. At the epoch of galaxy formation, gravitational instability develops on a variety of scales, which may lead to formation of small-scale dark matter clumps in galaxy halos. Dark matter particles trapped in a gravitational well are out of thermal equilibrium and are nonrelativistic. Typically interparticle interactions are very small, but, if the particles are bosons, the relaxation time can in certain cases be comparable to the age of the universe [6]. This opens a possibility of Bose-Einstein condensation and formation of Bose stars [7-9]. One proposed precursor of those is axion miniclusters [10], but modern particle models contain a variety of other fields of potential interest in this respect: majoron, dilaton, moduli, to name a few.
Thus, it is important to investigate the evolution of nonequilibrium Bose gases under various conditions. If the interaction between the particles is repulsive, and the energy density is sufficiently low, a Bose-Einstein condensate will form. The process of Bose-Einstein condensation in this case has been studied theoretically in a number of papers [6], [11-13].

The question we want to address in this paper is what happens to a nonequilibrium Bose gas if the interaction between its particles is attractive, at least within a certain range of interparticle distances. There is hardly any doubt that an attractive interaction will lead to clumping and phase separation, and statements to that effect have appeared in recent literature [14]. However, it has remained unclear whether the clumps will be in the normal or the phase-coherent (superfluid) state. In addition, kinetics of the clumping needs to be elucidated. Our main result, obtained via numerical integrations, is that the clumps are drops of phase-coherent quantum liquid (Bose-Einstein condensate), analogous to ${ }^{4} \mathrm{He}$. These drops remained suspended in uncondensed gas for as long as we could follow the evolution, although they did grow somewhat at the expense of the gas. The true ground state, which is one large drop, has not been reached in our simulations, and it may be unreachable in practice as well. Because the phase coherence of the drops is attributable to the quantum statistics of the particles, we call such drops quantum dew.

The coherent, macroscopically ordered nature of quantum dew may be important in cosmological (as well as laboratory) applications. Suppose for example that the particles it is made of can decay into some other particles. The macroscopically populated mode of a coherent clump may work as a laser $[8,15]$; as a result, quantum dew may decay much faster than an incoherent clump would.

The purpose of the present paper is to prove the coherent nature of the clumps and to study the kinetics of appearance and growth of dew drops. For this purpose, we have chosen the simplest model with an attractive interaction and, nevertheless, a stable ground state. From the point of view of cosmological applications, perhaps the most important effects left out of this simple model are the expansion of the 
universe and the gravitational attraction. The expansion dilutes gas available for clumping and thus slows the clumping down. The gravitational attractions works in the opposite direction. The net effect of these opposing tendencies can in principle be found via numerical integrations, and we plan to return to this important question in future.

\section{BOSE FIELD WITH ATTRACTIVE SELF-COUPLING}

\section{A. The model}

The model contains a nonrelativistic complex Bose field $\psi$ with the following equation of motion

$$
2 m i \frac{\partial \psi}{\partial t}=-\nabla^{2} \psi-|\psi|^{2} \psi+g_{6}|\psi|^{4} \psi
$$

The field is normalized so that the attractive cubic term on the right-hand side has the coefficient of unity. The corresponding coupling $g_{4}$ then appears in the commutation relation

$$
\left[a_{\mathbf{k}}, a_{\mathbf{k}^{\prime}}^{\dagger}\right]=\left|g_{4}\right| \delta_{\mathbf{k k}^{\prime}},
$$

the annihilation operators $a_{\mathbf{k}}$ being defined via $\psi(\mathbf{r})$ $=V^{-1 / 2} \Sigma_{\mathbf{k}} a_{\mathbf{k}} \exp (i \mathbf{k r})$ in a finite volume $V$. The quintic term in Eq. (1) is repulsive, and it becomes important when $\psi^{\dagger} \psi$ approaches $g_{6}^{-1}$. [The coupling $g_{4}$ appearing in Eq. (2) is related to $\lambda$ of the relativistic $\lambda \phi^{4} / 4$ potential via $g_{4}$ $=3 \lambda / 2 \mathrm{~m}$, and to the scattering length $a$ of a nonrelativistic Bose gas via $g_{4}=8 \pi a$; here $\hbar=1$. The physical density is $\left.\psi^{\dagger} \psi /\left|g_{4}\right|.\right]$

\section{B. Initial conditions}

Our integrations are set up as follows. In the initial state the occupation numbers $n_{\mathbf{k}}=a_{\mathbf{k}}^{\dagger} a_{\mathbf{k}}$ have a Gaussian distribution over momenta

$$
n_{\mathbf{k}}=A \exp \left(-k^{2} / k_{0}^{2}\right)
$$

The population of the homogeneous mode is $n_{0}=A$, which is not considerably larger than population of other modes with small $k$. In this sense, there is no macroscopic condensate in the initial state.

\section{Classical approximation}

Now, we assume that $g_{4}$ in Eq. (2) is small compared to $A$ in Eq. (3). Then, we can neglect the commutator of $a$ and $a^{\dagger}$ compared to the typical magnitude of $a$ itself, see Bogoliubov [1]. As a result, the problem becomes classical and can be integrated on a lattice. This classical approximation has been used to study nonlinear dynamics of relativistic Bose fields at large occupation numbers [16] and the process of Bose-Einstein condensation [13]. In the present work, we use it to demonstrate the formation of quantum dew in the model (1). This use involves no contradiction of terms: quantum dew is an effect of quantum statistics when one thinks in terms of individual particles, but it comes out as an effect of classical evolution in the collective, field-theoretical descrip- tion. Notice that Eq. (3) determines only absolute values of $a_{\mathbf{k}}$; their phases are chosen as uncorrelated random numbers.

\section{Physical scales}

We choose the parameters of the model in such a way that $\left\langle\psi^{\dagger} \psi\right\rangle \ll g_{6}^{-1}$; the angular brackets denote averaging over the lattice. This means that in the initial state the attractive interaction is much more important than the repulsive one. In this case, we expect that, in appropriate dimensionless units, the time scale $t_{c}$ of the initial collapse of the gas into clumps depends only on the single remaining parameter of nonlinearity

$$
\xi=\frac{\left\langle\psi^{\dagger} \psi\right\rangle}{2 m \epsilon},
$$

where $\epsilon$ is the average kinetic energy per particle in the initial state; $\xi$ is of order of the ratio of the initial potential energy of attraction to the initial kinetic energy. A similar parameter for an atomic gas in a trap will be introduced below. We can write

$$
t_{c}^{-1}=\epsilon F(\xi),
$$

where $F$ is some function obeying the condition $F(0)=0$ and $F(1) \sim 1$. The form of $F(\xi)$ at small $\xi$ is established below. For the initial distribution (3), the initial parameter of nonlinearity is $\xi=k_{0} A / 12 \pi^{3 / 2}$. We choose units of time so that $2 m=1$. We also use units of length in which $k_{0}=2 \pi$, i.e., measure lengths in units of the particles' typical initial de Broglie wavelength. Except where stated otherwise, we consider the case of moderate nonlinearity $A=5$, which corresponds to $\xi=0.47$. We use $g_{6}^{-1}=3600$.

\section{NUMERICAL RESULTS}

\section{A. Numerical procedure}

The results below are from integrations on a $64^{3}$ cubic lattice with side $L=2.25$ (in the above length units) and periodic boundary conditions. The state of the system was updated via a second-order in time algorithm based on the Crank-Nicholson method for the diffusion equation. The algorithm conserves the number of particles exactly. Energy nonconservation was below $2 \%$ for the entire integration time.

\section{B. Formation of dew}

Figure 1 shows two snapshots of the field, at times $t$ $=0.1$ and $t=20$. Dots have been placed on all lattice sites at which $|\psi|>30$ (the mean-square value of $|\psi|$ is 5.3). Drops of dew are clearly seen.

A movie of the evolution of this picture from $t=0.1$ to $t$ $=20$ shows that the drops of dew move around, gradually slowing down, and occasionally coalesce. The overall growth of the number of sites with $|\psi|>30$ continues even at $t=20$, the latest time in our computation, but at that time it is already quite slow. If we define that grid points with $|\psi|$ $\leqslant 8$ belong to the gas, and correspondingly grid points with 


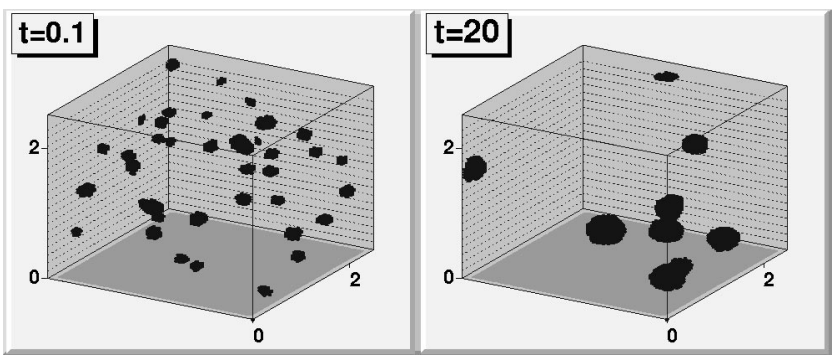

FIG. 1. Drops of dew at different moments of time.

$|\psi|>8$ belong to the dew $(|\psi|=8$ is approximately the boundary between the gas and the dew at $t=20$, see Fig. 3 below), we find that around $15 \%$ of all particles are in the gas, and around $85 \%$ had condensed in the dew by the time $t=20$. These fractions, however, may be altered when gravity is included.

Figure 2 shows initial stages of the condensation process: we plot the fraction of particles that are in the dew, as a function of time. We observe two distinct stages: a rapid collapse followed by a slower evolution. Because $\xi \sim 1$ at $A=5$, we estimate the time of collapse $t_{c}$ from Eq. (5) as $t_{c} \sim k_{0}^{-2}$. For $k_{0}=2 \pi$ this gives $t_{c} \sim 0.025$, in good agreement with the data of Fig. 2. In the regime of weak nonlinearity, $\xi \ll 1$, we expect the collapse to be due to two-particle collisions, in which case $F(\xi) \propto \xi^{2}$. [With this form of $F(\xi)$, the estimate Eq. (5) for the time of the collapse coincides with the estimate of the condensation time of Ref. [6], which can also be obtained from a solution to the Boltzmann equation [12]]. The time of the collapse then has to scale as $A^{2}$ when we decrease $A$ and keep all other parameters fixed. Results of integrations with different values of $\xi<1$ confirm this, see Fig. 2.

\section{Cold atomic gases}

For an atomic gas confined in a trap, at some temperature $T$, one can introduce initial parameter of nonlinearity $\xi_{T}$ :

$$
\xi_{T}=\frac{4 \pi \hbar^{2}|a| n}{m T},
$$

where $n$ is a typical gas density, and $a$ is the scattering length, which in the present case is negative. In Eq. (6), $k_{B}$ $=1$, but we have restored $\hbar$. Let us use for estimates $n$ $=\left(m T_{0} / 3.31 \hbar^{2}\right)^{3 / 2}$ and $T=T_{0}$, where $T_{0}$ is the temperature of BEC of an ideal monoatomic gas in a given trap and with a given number of particles. Bradley et al. [3] quote $T_{0}$ $=300 \mathrm{nK}$ and $a=-27.3 a_{0}$ for their experiment with trapped ${ }^{7} \mathrm{Li}$ ( $a_{0}$ is the Bohr radius); using these values we obtain $\xi_{T}=0.006$. Estimating the rate of the collapse as $\hbar t_{c}^{-1} \sim T_{0} \xi_{T}^{2}$, we find $t_{c} \sim 1 \mathrm{~s}$. We thus expect that quantum dew can be observed in laboratory in traps of a sufficiently large size.

\section{Field distribution}

The onset of the slower evolution indicates that a chemical quasiequilibrium between the dew and the gas has been

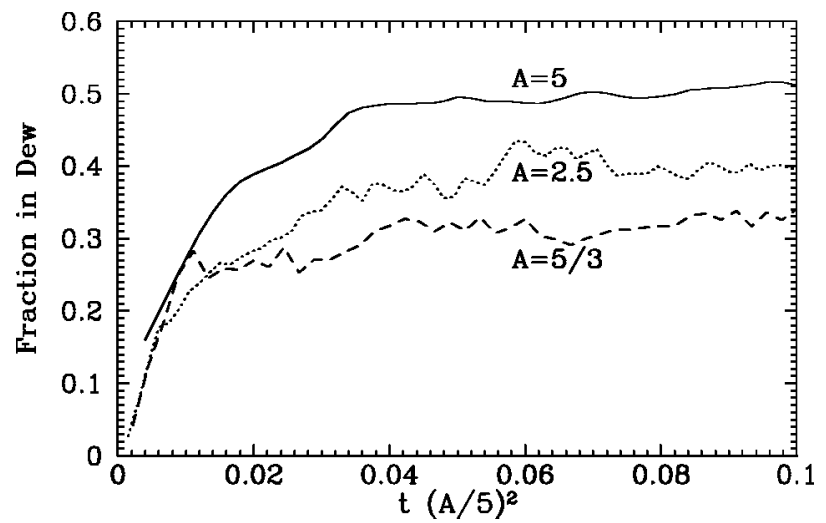

FIG. 2. Condensation process.

reached, i.e., the processes of evaporation of particles from the existing dew drops and condensation back onto them are approximately (but not exactly) balanced. This interpretation is supported by the following test. The probability distribution of the absolute value of the field over lattice sites shows two distinct peaks: one at large $|\psi|$, corresponding to the dew drops, and another at small $|\psi|$, corresponding to the gas of particles, see Fig. 3. If at some instant we remove the gas, i.e. set $\psi=0$ at all sites where we had $|\psi|<10$, and then continue the evolution, the gas reappears, while the number of sites occupied by the dew decreases down to another slowly evolving value. Apparently, the dew partially evaporates, so as to restore the chemical quasiequilibrium.

Finally, Fig. 4 illustrates the coherent nature of the dew. It shows the field $\psi$ at $t=0.2$ at a section of our integration cube parallel to the $x-y$ plane. For visual clarity only eveneven numbered sites are included. The length of an arrow represents $|\psi|$, and the angle clockwise from 12 noon represents $\arg \psi$. We see that the sites occupied by the dew (i.e., having large $|\psi|$ ) are in drops, and each such drop is coherent- the arrows point approximately in the same direction. The direction of arrows in each drop rotates with time, just as in the homogeneous case [1], but these directions are different for different drops. Similar slices at later times show that the clumping becomes more pronounced, the dew

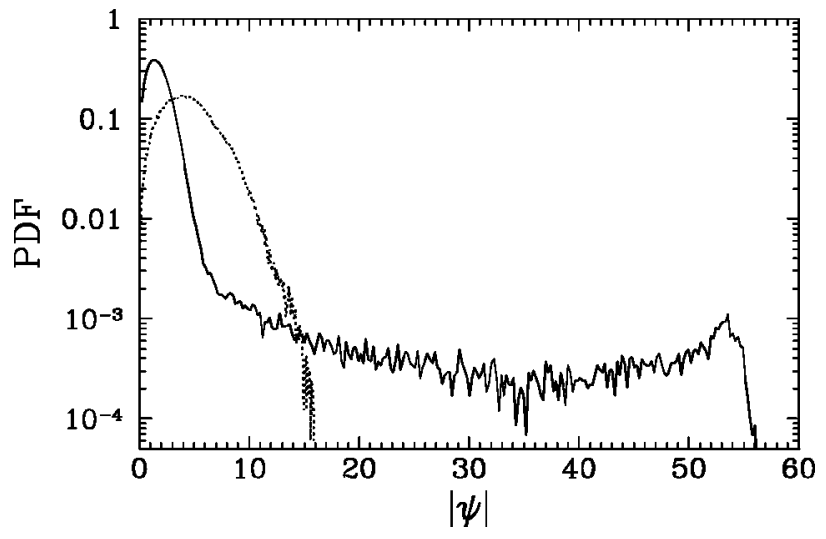

FIG. 3. Probability distribution function of the field magnitude at $t=20$ (the solid line). Initial probability distribution is shown by the dotted line. 


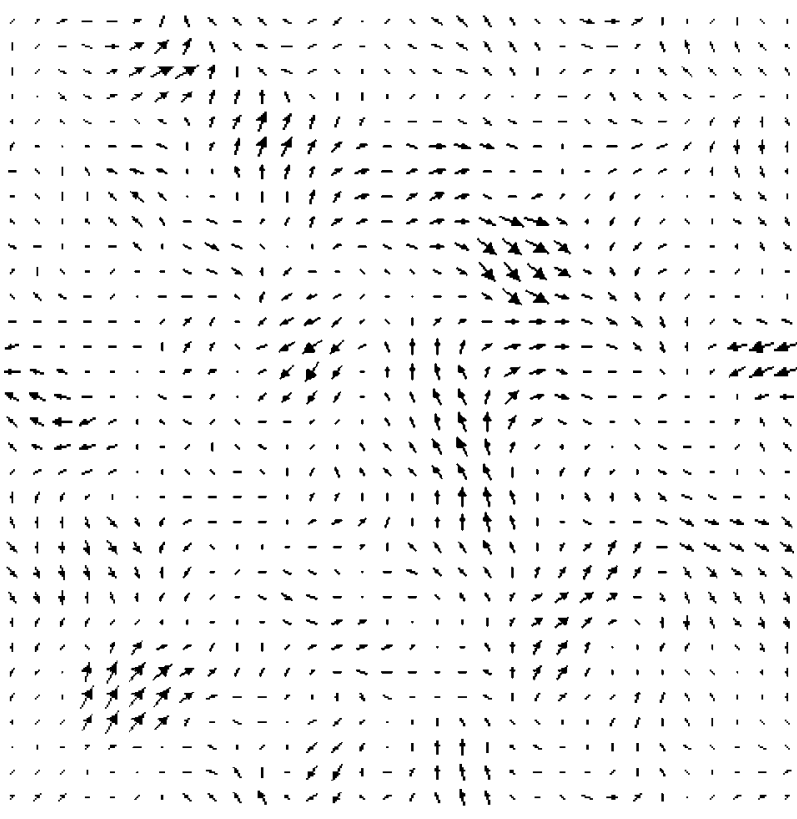

FIG. 4. Field distribution in a spatial slice at $t=0.2$.

is still coherent, while the remaining gas (occupying sites with small $|\psi|$ ) is incoherent.

\section{E. Dew and $Q$ balls} form

Equation (1) has stable nontopological solitons of the

$$
\psi(\mathbf{r}, t)=\chi(\mathbf{r}) \exp (i \omega t)
$$

(nonrelativistic analogues of $Q$ balls [17]). As we continue to truncate the gas, i.e., to remove particles from sites with progressively smaller $|\psi|$, and to evolve the system between these truncations, we expect to eventually reach a state in which solitons float in vacuum (or gas of a very small density). Changes in the probability distribution function (PDF) of $|\psi|$ resulting from this procedure are shown in Fig. 5. We interpret the limiting form to which the PDF converges in the middle range of $|\psi|$ as corresponding to the wall profile of nontopological solitons. Computation of $\psi$ at the center of a

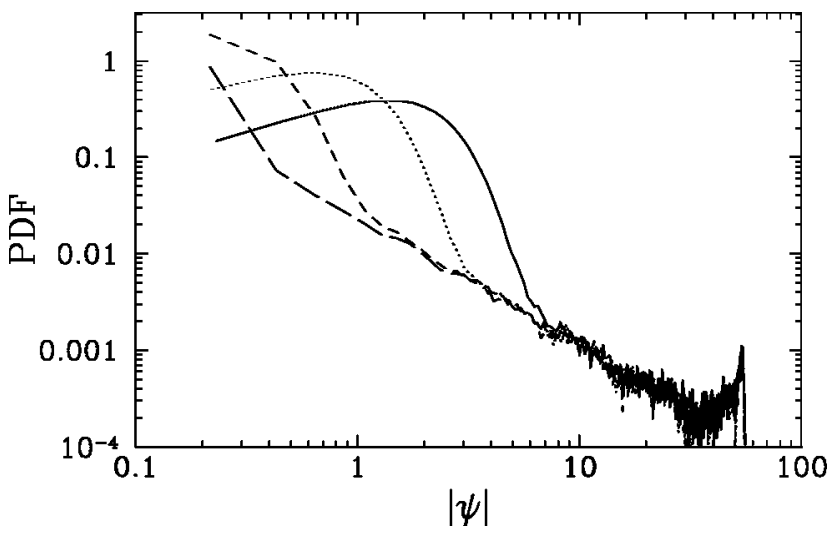

FIG. 5. PDF of the field's magnitude after truncating the gas and letting it reequilibrate. Solid line: no truncation; dotted line: after truncation at $\psi=10$; dashed line: after further truncation at $\psi=3$; long dashed: after further truncation at $\psi=1$.

soliton in the thin-wall approximation gives $|\psi|_{c}$ $=\left(3 / 4 g_{6}\right)^{1 / 2} \approx 52$, in good agreement with the position of the peak in the PDF at large $|\psi|$. Like nontopological solitons produced in a decay of an unstable homogeneous condensate [18], quantum dew may work as cold dark matter.

\section{CONCLUSION}

To summarize, our main results are (i) a numerical proof that the clumps of matter formed in a nonequilibrium gas with an attractive interaction are drops of coherent quantum liquid (Bose-Einstein condensate); (ii) evidence that the rapid collapse of particles into drops of this quantum dew is followed by a slower evolution, during which the dew is in approximate chemical equilibrium with the surrounding gas; (iii) evidence that at weak nonlinearity the rate of the initial collapse is consistent with being determined by two-particle collisions.

\section{ACKNOWLEDGMENTS}

We thank A. Kusenko and M. Shaposhnikov for useful discussions. This work was supported in part by U.S. DOE Grant No. DE-FG02-91ER40681 (Task B) and NSF Grant No. PHY-9501458. S.K. thanks ITP, Santa Barbara for hospitality during completion of this work.
[1] N. N. Bogoliubov, J. Phys. (Moscow) 11, 23 (1947).

[2] M. H. Anderson et al., Science 269, 198 (1995).

[3] C. C. Bradley, C. A. Sackett, J. J. Tollett, and R. G. Hulet, Phys. Rev. Lett. 75, 1687 (1995).

[4] K. B. Davis et al., Phys. Rev. Lett. 75, 3969 (1995).

[5] S. Khlebnikov and I. Tkachev, Phys. Rev. Lett. 79, 1607 (1997); S. Khlebnikov, L. Kofman, A. Linde, and I. Tkachev, ibid. 81, 2012 (1998).

[6] I. I. Tkachev, Phys. Lett. B 261, 289 (1991).

[7] R. Ruffini and S. Bonozzola, Phys. Rev. 187, 1767 (1969); for a review see A. R. Liddle and M. S. Madsen, Int. J. Mod. Phys. D 1, 101 (1992).

[8] I. I. Tkachev, Sov. Astron. Lett. 12, 305 (1986).
[9] M. Colpi, S. L. Shapiro, and I. Wasserman, Phys. Rev. Lett. 57, 2485 (1986).

[10] E. W. Kolb, I. Tkachev, Phys. Rev. Lett. 71, 3051 (1993); Phys. Rev. D 49, 5040 (1994); Astrophys. J. Lett. 460, L25 (1996).

[11] E. Levich and V. Yakhot, J. Phys. A 11, 2237 (1978); B. V. Svistunov, J. Mosc. Phys. Soc. 1, 373 (1991); H. T. C. Stoof, Phys. Rev. Lett. 66, 3148 (1991); Yu. M. Kagan, B. V. Svistunov, and G. V. Shlyapnikov, Zh. Éksp. Teor. Fiz. 101, 528 (1992) [Sov. Phys. JETP 74, 279 (1992)].

[12] D. V. Semikoz and I. I. Tkachev, Phys. Rev. Lett. 74, 3093 (1995); D. V. Semikoz and I. I. Tkachev, Phys. Rev. D 55, 489 (1997). 
[13] K. Damle, S. N. Najumdar, and S. Sachdev, Phys. Rev. A 54, 5037 (1996).

[14] H. T. C. Stoof, Phys. Rev. A 49, 3824 (1994); C. A. Sackett, H. T. C. Stoof, and R. G. Hulet, Phys. Rev. Lett. 80, 2031 (1998)

[15] I. I. Tkachev, Phys. Lett. B 191, 41 (1987).

[16] S. Khlebnikov and I. Tkachev, Phys. Rev. Lett. 77, 219 (1996).
[17] G. Rosen, J. Math. Phys. 9, 996 (1968); 9, 999 (1968); R. Friedberg, T. D. Lee, and A. Sirlin, Phys. Rev. D 13, 2739 (1976); S. Coleman, Nucl. Phys. B262, 263 (1985).

[18] A. Kusenko and M. Shaposhnikov, Phys. Lett. B 418, 46 (1998); M. Laine and M. Shaposhnikov, Nucl. Phys. B532, 376 (1998). 